\title{
Holistic Reasoning on Quality of Life Dimensions Based on Mobile apps with Ontology
}

\author{
Isabela N. Cavaco ${ }^{1}$, Clauirton A. Siebra ${ }^{1}$ \\ ${ }^{1}$ Centro de Informática - Universidade Federal da Paraíba (UFPB) \\ João Pessoa - PB - Brazil \\ \{inc@cin.ufpe.br, clauirton@ci.ufpb.br\}
}

\begin{abstract}
To integrate the information about diverse QoL dimensions and enable the specification of a holistic process of reasoning that can improve the effectiveness of interventions, we explore the integration of different $Q o L$ aspects in the form of an ontological model, presenting associations between their concepts that should be considered together when inferences are made, since they are usually intrinsically related and would not be complete when separately analysed. These dimensions will be studied from a selected bundle of iOS and Android mobile apps within QoL aspects of Diet, Emotional, Physical Exercises and Physical Health (e.g. Pregnancy, Blood Pressure and Sleep).
\end{abstract}

\section{General Information}

The growth of mobile applications solving everyday problems has lead smartphone users to increasingly look for technological solutions to take better care in health and get assessments to keep them in the right path, improving their Quality of Life (QoL). As defined by the World Health Organization (WHO), QoL is the individual's perceived position in life, in the context of the culture and value systems in which they live and in relation to their goals and expectations, standards and concerns. The QoL is divided in 4 domains (Physical Health, Psychological Health, Social relation and Environment). As a means to assess an individual's QoL, WHO has created a scale called WHOQOL divided into 24 subdimensions (see Figure 1), which can be measured using specific instruments.

Considering the QoL subject, many mobile applications exist to support people's needs, and smartphones are close to their users at least half of the time [Wac et al. 2015a]. The authors also mention that smartphones and wearables are likely the best tools to help health improvement and QoL in everyday activities. According to Wac (2018), it was estimated that $60 \%$ of the US population in 2013 tracked some aspect of their life (e.g., weight, exercise, mood), 33\% of adults tracked health indicators or symptoms (e.g., blood pressure, blood sugar, headaches, or sleep patterns), and $12 \%$ tracked a health indicator on behalf of someone they cared for. As stated by Ciman and Wac (2016), we're always in stressful situations, specially in the past few decades, negatively influencing people's lives in the long term. Much can be said about this topic, but as affirmed by Lane and Georgiev (2015), "with the use of sensors in wearables and smartphones, apps are giving users new powerful mobile experiences that have the potential to change the way users live and interact with each other", offering 
interventions and plans of action to improve QoL. According to the authors, users can quantify their sleep and exercise patterns, monitor personal commute behaviors, track their emotional state, and much more.

The QoL research often involves knowledge representation in the form of ontology, which was defined by Snae and Brüeckner (2007) as a philosophic word that means explicit specification of a conceptualization, e.g., representing conceptual descriptions of the specific content as they assist to identify appropriate items and relationships in a given knowledge domain. Furthermore, according to the authors, ontologies show a hierarchical dependence of the terms together with descriptions, explanations and definitions. Consider an app for physical activity intervention that can indicate a QoL programme for losing weight. The studies of Miller, Koceja and Hamilton (1997) and Caudwell et al. (2009) demonstrated that even when exercise energy expenditure is high, a healthy diet is still required for weight loss to occur in many people. Thus, the assessment of the physical activity and nutritional dimensions should be conducted together. Unfortunately, this may not still be enough, because even if individuals have a good behaviour in both dimensions, a stressful life associated with psychological conditions, liberates an extra amount of cortisol that signals the body to shift metabolism to store fat. Thus, all of these dimensions should be evaluated together so that interventions should not be individually delivered to each specific issue. In order to integrate the information about diverse QoL dimensions and enable the specification of a holistic process of reasoning that can improve the effectiveness of interventions, this work proposes to integrate these different QoL dimensions in the form of an ontological model, thus presenting associations between concepts of different dimensions that should be considered together when inferences are made, since they are usually intrinsically related and would not be complete by analysing separately, as exemplified before.

The current state of the art brings several proposals and applications for mobile assessment and interventions by means of mobile devices. An example is the work of Fallaize (2019), which evaluates the agreement level of popular nutrition-related apps for the energy and available macronutrients and micronutrients assessment against a UK reference method. In a different domain, the work of Ciman and Wac (2016) presents an approach for stress assessment that leverages data extracted from smartphone sensors, and that is not invasive concerning privacy. There are also approaches for physical activities [Peart, Balsalobre and Shaw 2019] and sleeping patterns [Burgdorf 2018] assessment. However, Wac and colleagues indicate that "there is no holistic app for researchers and smartphone users to deploy and participate in evidence-based longitudinal, multidimensional studies, generating high-resolution datasets to assess and then change behaviours and improve QoL in the long-term" [Manea and Wac 2018; Wac et al. 2015b]. Thus, we exploit the development of an integrated ontology model and related holistic reasoning processes as a differential that could provide the mobile development a powerful resource to create better interventions based on the multidimensional assessment of individuals' QoL parameters. To achieve this goal, we have selected a bundle of iOS and Android mobile apps within QoL dimensions of Diet, Emotional, Physical Exercises and Physical Health (e.g. Pregnancy, Blood Pressure and Sleep), analysing each and every app required data inputs in order to design the ontological model, as we'll discuss in the next sections. 
The remainder of this paper is organized as follows. Section 2 define specifies the methodology of this research. Section 3 presents the ontology model with the proposed dimensions designed with Protegé (ontology-editing environment). Lastly, Section 4 concludes this study with discussion and research directions.

\section{Methodology}

To achieve this work, a background study was conducted to review the current applications that can enable and help to improve the human Quality of Life (QoL), recognizing the state of the art for this subject, regarding the advantages and strengths, disadvantages and difficulties, amongst other information that may be considered relevant, by defining some research questions described next.

For the research, 120 applications for Android and iOS platforms were selected, being divided into 12 theme groups, such as Physical Health, Health care, Emotional and Social, among others.

For each group, 10 applications were selected (5 for each operating system Android and iOS). After the selection of these apps, they have been analysed in terms of data input required from users, such as age, current weight, frequency of exercises, and others. With the information collected from this analysis and the subdomains of Diet, Physical Health, Health care and Emotional, we began to develop the ontology model using Protegé editing environment, following the steps provided by Noy and McGuinness (2001) in their guide to creating ontology models.

\section{Ontology Model}

As defined by Gruber (1993), ontology is an explicit formal specification of the terms in the domain and relations among them, and has been widely used in the field of Artificial Intelligence. According to Noy and McGuinness (2001), an ontology defines a common vocabulary for researchers who need to share information in a domain.

Conforming to Noy and McGuinness (2001), some of the reasons to develop ontology are:

- Share common understanding of the information structure among people or software agents;

- Enable the reuse of domain knowledge;

- Make domain assumptions explicit;

- Separate domain knowledge from the operational knowledge;

- Analyze domain knowledge.

The authors exemplify and explain each of the above in detail, as their work is a guide to constructing ontology models using Protegé-2000 editing environment.

To construct the model, we've analysed the user input requested by the 40 applications. The relationship between the domain areas is related to the similar information that is requested as data entry in the selected mobile applications. 
Therefore, we have first identified information related to the basic personal users profile and defined the following variables as part of the Person class: age, gender, weight and height.

An important aspect is how emotional-related areas, for example, can have an effect on the diet or how a person faces an exercise routine. Factors such as stress and anxiety can lead the user to eat in an uncontrolled way or even impact the quality of sleep, a factor that also relates to another area called as Physical Condition. This area contains applications that seek for measuring blood pressure, heartbeat, sleep quality, breathing, among others. The emotional aspect was divided into the subclasses GoodEmotionalState and BadEmotionalState, both of which impact the whole context of a healthy life, which is the main objective of QoL applications. The next schema (Figure 2) illustrate part of the model as way to exemplify some of these relationships.



Figure 1. Sample of the holistic QoL model using Ontology

Some base classes were also included in our model, such as Meal and Body. Meal includes the type of meal, food group and nutrients. The body includes parts of the body that are part of Exercise Definition, where it contains types of exercises according to the main areas to be impacted.

When it comes to Physical Exercises, usually a Diet Objective is involved, which could be losing, maintaining or increasing weight, or even to build muscle mass, that defines the Type of Exercise that needs to be performed to achieve it. A person's Motivation will have an impact of controlling the Diet, affecting the latter's initial state, in alignment with the Diet Goal and affecting the Exercise Frequency.

These examples show the interconnection of areas, and the importance to understand each aspect of QoL as a unique set, representing the context in which we're inserted when we look for a healthy life.

\section{Discussion and future work}


The motivation to work with ontology within the previously mentioned QoL subdomains was to create models that support holistic forms of reasoning. Then, the first part of this work was to identify the QoL concepts that are considered by the current mobile applications and create a unique knowledge representation que relates such concepts. This work presents the first version of this ontology, which will be extended with other concepts and rules representing the health relations concerning the QoL dimensions. This strategy is different from other current approaches, which assess individual dimensions and do not provide holistic forms to analyse the QoL of users.

The difficulties encountered until the moment are related to the analysis of some app features, which require their purchase/subscription. Consequently, we are only focusing our research on free apps, which can limit our view about the type of information that we can in fact obtain by means of this technology.

As future work, we intend to conclude the ontology model and formulate its relations in more detail. Then, we intend to evaluate the final ontology model by following two strategies that are focused on different set of criteria and are commonly used to evaluate such representations, as better detailed in [Degbelo 2017].

\section{References}

Achananuparp, P., Lim, E. P. and Abhishek, V. (2018) "Does journaling encourage healthier choices? Analyzing healthy eating behaviors of food journalers". In Proceedings of the 2018 International Conference on Digital Health (pp. 35-44).

Burgdorf, A., Güthe, I., Jovanović, M., Kutafina, E., Kohlschein, C., Bitsch, J. Á. and Jonas, S. M. (2018) "The mobile sleep lab app: An open-source framework for mobile sleep assessment based on consumer-grade wearable devices". Computers in biology and medicine, 103, 8-16.

Caudwell, P., Hopkins, M., King, N. A., Stubbs, R. J., \& Blundell, J. E. (2009) "Exercise alone is not enough: weight loss also needs a healthy (Mediterranean) diet?". Public health nutrition, 12(9A), 1663-1666.

Ciman, M. and Wac, K. (2016) 'Individuals' stress assessment using human-smartphone interaction analysis". IEEE Transactions on Affective Computing, 9(1), 51-65.

Degbelo, A. (2017) “A snapshot of ontology evaluation criteria and strategies". In Proceedings of the 13th International Conference on Semantic Systems (pp. 1-8).

Fallaize, R., Franco, R. Z., Pasang, J., Hwang, F., \& Lovegrove, J. A. (2019) "Popular Nutrition-Related Mobile Apps: An Agreement Assessment Against a UK Reference Method". JMIR mHealth and uHealth, 7(2), e9838.

Gill, T. M. and Feinstein, A. R. (1994) "A critical appraisal of the quality of quality-of-life measurements”. Jama, 272(8), 619-626.

Gruber, T. R. (1993) "A translation approach to portable ontology specifications". Knowledge acquisition, 5(2), 199-221. 
Lane, N. D. and Georgiev, P. (2015) “Can deep learning revolutionize mobile sensing?", In Proceedings of the 16th International Workshop on Mobile Computing Systems and Applications (pp. 117-122).

Manea, V. and Wac, K. (2018) "mQoL: mobile quality of life lab: from behavior change to QoL". In Proceedings of the 2018 ACM International Joint Conference and 2018 International Symposium on Pervasive and Ubiquitous Computing and Wearable Computers (pp. 642-647).

Martinez, P. M. (1998) “An introduction to the concept of "quality of life in Parkinson's disease".” Journal of neurology, 245(1), S2-S6.

Miller, W. C., Koceja, D. M. and Hamilton, E. J. (1997) "A meta-analysis of the past 25 years of weight loss research using diet, exercise or diet plus exercise intervention." International journal of obesity, 21(10), 941-947.

Noy, N. F. and McGuinness, D. L. (2001) "Ontology development 101: A guide to creating your first ontology".

Park, S. and Jayaraman, S. (2003) "Enhancing the quality of life through wearable technology". IEEE Engineering in medicine and biology magazine, 22(3), 41-48.

Peart, D. J., Balsalobre, C. F. and Shaw, M. P. (2019) "Use of mobile applications to collect data in sport, health, and exercise science: a narrative review." The Journal of Strength \& Conditioning Research, 33(4), 1167-1177.

Snae, C. and Brüeckner, M. (2007) "Ontology-driven e-learning system based on roles and activities for Thai learning environment". Interdisciplinary Journal of E-Learning and Learning Objects, 3(1), 1-17.

Wac, K. (2018) "From quantified self to quality of life". In Digital Health (pp. 83-108). Springer, Cham.

Wac, K. et al. (2015a) "Quality of life technologies: Experiences from the field and key challenges". IEEE Internet Computing, 19(4), 28-35.

Wac, K. et al. (2015b) "mQoL: Experiences of the 'Mobile Communications and Computing for Quality of Life'", Living Lab, 10.1109/HealthCom.2015.7454494. 